\title{
The Midline Sagittal View of Fetal Brain: Moving from 3D to 2D
}

\author{
Ladbans Kaur $^{1}$ (D) $\cdot$ Divya Singh ${ }^{1} \cdot$ Meetan Preet Kaur ${ }^{1}$
}

Received: 17 August 2015/Accepted: 13 November 2015/Published online: 10 December 2015

(C) Society of Fetal Medicine 2015

\begin{abstract}
The objective of the present study was to investigate the effectiveness of a simplified approach by using two dimensional (2D) technique for evaluation of the midline structures of the fetal brain and comparing it with the three dimensional (3D) technique. The study was performed in 60 fetuses with normal intracranial anatomy and 10 fetuses with anomalies involving the midline cerebral structures from 19 to 28 weeks of gestation. Ultrasound examinations were performed on Voluson E8 BT10. 2D median planes were obtained by aligning the transducer through the anterior and posterior fontanelle and the sutures by either transabdominal sonogram or transvaginal sonogram (TVS) approach. Median planes were also obtained by 3D. Visualization of the relevant anatomic details, corpus callosum and cerebellar vermis in particular, was noted by both $2 \mathrm{D}$ and 3D images. 2D and 3D median planes were compared qualitatively. 3D midline sagittal planes could be visualized in 48/50 normal fetuses. 2D median planes were obtained in all, usually more easily and rapidly. TVS was also used in fetuses with breech presentation. There was a good correlation between 2D and 3D images. The abnormal group included 10 fetuses. In all cases, the diagnosis could be made by both $2 \mathrm{D}$ and $3 \mathrm{D}$ views but 3D added clinically useful information especially in two fetuses with posterior fossa lesions. The 2D median views yielded excellent display of anatomy as well as the abnormalities. With high resolution probes, 2D median planes are good enough which can substitute 3D median plane views, especially in developing countries where there is less availability of $3 \mathrm{D}$ equipment and expertise of the 3D technique.
\end{abstract}

Ladbans Kaur

ladbans@gmail.com

1 Prime Diagnostic Centre and Heart Institute, S.C.O. 155, Sector-24D, Chandigarh 160023, India
Keywords Fetal brain - Three-dimensional ultrasound . Corpus callosum $\cdot$ Vermis

\section{Introduction}

The development of fetal brain is a complex mechanism producing remarkable changes in its sonographic appearance throughout pregnancy. The imaging of fetal brain begins by evaluating the three standard planes [1]. The first plane passes through the cavum septum pellucidum and the atria of the lateral ventricles (transventricular plane), the second through the cavum septum pellucidum and the thalami (transthalamic plane), and the third passes through the posterior fossa and the cerebellum (transcerebellar plane). In fact, the evolution of three dimensional (3D) ultrasound has given a new dimension of looking at fetal brain. With this background knowledge of anatomy on 3D, these planes can be obtained on two dimensional (2D) ultrasound with better resolution. The study was performed to evaluate the effectiveness of a simplified approach by using 2D technique for evaluation of the midline structures of the fetal brain and also comparing it with the $3 \mathrm{D}$ technique. To improve upon the detection rate, a combination of sagittal, coronal, parasagittal, and angled views on 2D imaging to visualize the midline structures was used [2].

\section{Materials and Methods}

This was a prospective study of 60 consecutive pregnancies at 19-28 weeks of gestation. Ultrasound examination was performed by using Voluson E8 ultrasound system (GE healthcare) with $4-8 \mathrm{MHz}$ transabdominal probe and 
8-12 MHz transvaginal probe. 3D volume acquisitions were obtained. 2D median planes were obtained by aligning the transducer through the anterior and posterior fontanelle and the sutures. Both transabdominal and transvaginal routes were used to assess the midline structures depending upon the position of the fetal head. Of the 60 cases included in the present study, 50 fetuses showed normal intracranial anatomy. Ten fetuses had anomalies involving the midline cerebral structures.

On 2D imaging, direct visualization of corpus callosum was performed both on sagittal and coronal views. The length and the thickness of the corpus callosum were measured. In the posterior fossa, the vermis was also seen in both axial and midsagittal plane. The supero-inferior dimension of vermis was documented.

Median planes were also obtained by 3D technique [3]. Visualization of the relevant anatomic details of the corpus callosum and cerebellar vermis in particular, was noted by both $2 \mathrm{D}$ and $3 \mathrm{D}$ imaging and then these images were compared qualitatively.

\section{Results}

Three dimensional midline sagittal plane could be visualized in 48 out of 50 normal fetuses. 2D median plane was obtained in all, usually more easily and rapidly. There was a good correlation between 2D and 3D images. The abnormal group included the following cases. A fetus at 20 weeks showed nondelineation of cavum septum pellucidum on 2D transabdominal sonogram (TAS). On $3 \mathrm{D}$, corpus callosum was not visualized. 2D transvaginal sonogram (TVS) showed partial agenesis of corpus callosum with altered course of anterior cerebral artery ( Fig. 1a-c) [4-6].

Another patient was referred at 28 weeks' gestation for mild ventriculomegaly. Ultrasonography showed ventriculomegaly with normal length of corpus callosum, but there was thinning of the body and splenium of corpus callosum suggestive of hypoplasia. The fetus also showed post-axial polydactyly and receding chin. These features are consistent with a diagnosis of acrocallosal syndrome (Fig. 1d-g) [7].

A 26-year-old second gravida was referred for multiple congenital anomalies at 26-27 weeks. Biparietal diameter (BPD) was $<2$ nd centile by a TAS. 3D scan of the fetal head could not be performed due to fixed fetal position (distal arthrogryposis). So, a transvaginal 2D ultrasonography was done which yielded panorama of information showing features of ventriculitis, lissencephaly, widened subarachnoid space, periventricular calcification and leucomalacia, agenesis of the corpus callosum, bilateral cataractous changes, and lid edema (Fig. 2a-c) [8].

A case of septo-optic dysplasia at 24 weeks' was referred for mild ventriculomegaly. Imaging showed distinct absence of the cavum septum pellucidum. 3D TVS clearly demonstrated the presence of corpus callosum (Fig. 2d-f) [9].

Another patient with the history of consanguinity, presented at 28 weeks with fused and underdeveloped frontal
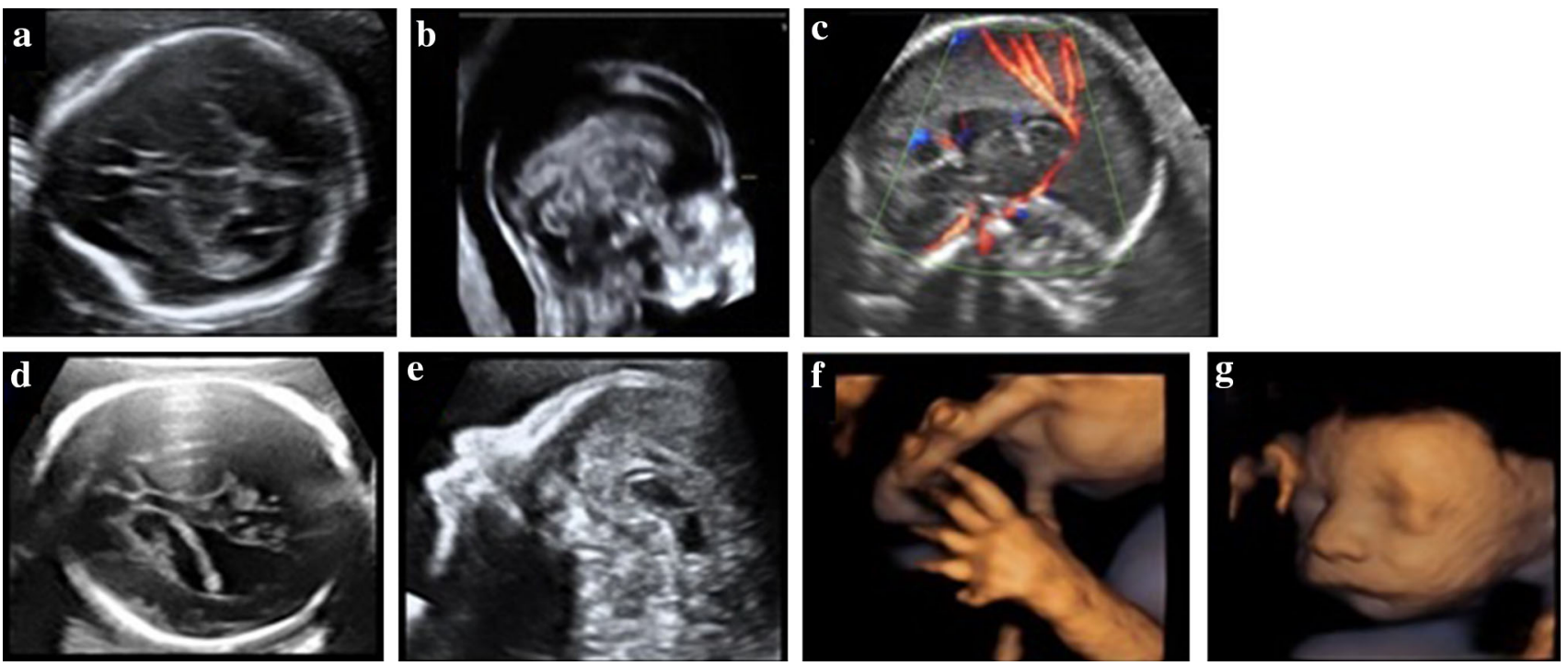

Fig. 1 a Partial agenesis of corpus callosum. TAS of fetal head at 20 weeks showing nondelineated cavum septum pellucidum b 3D volume contrast image (VCI) showing nonvisualization of corpus callosum c Transvaginal power angio image of the same case with a deviated course of anterior cerebral artery-'snake under the

table sign'. Note partial visualization of rostrum and genu of corpus callosum d Acrocallosal syndrome. Sonography of 28 weeks fetus showing borderline ventriculomegaly e Sagittal section showing reduced thickness of the body and splenium of corpus callosum $\mathbf{f} 3 \mathrm{D}$ rendered image showing post-axial polydactyly $\mathbf{g}$ Micrognathia 

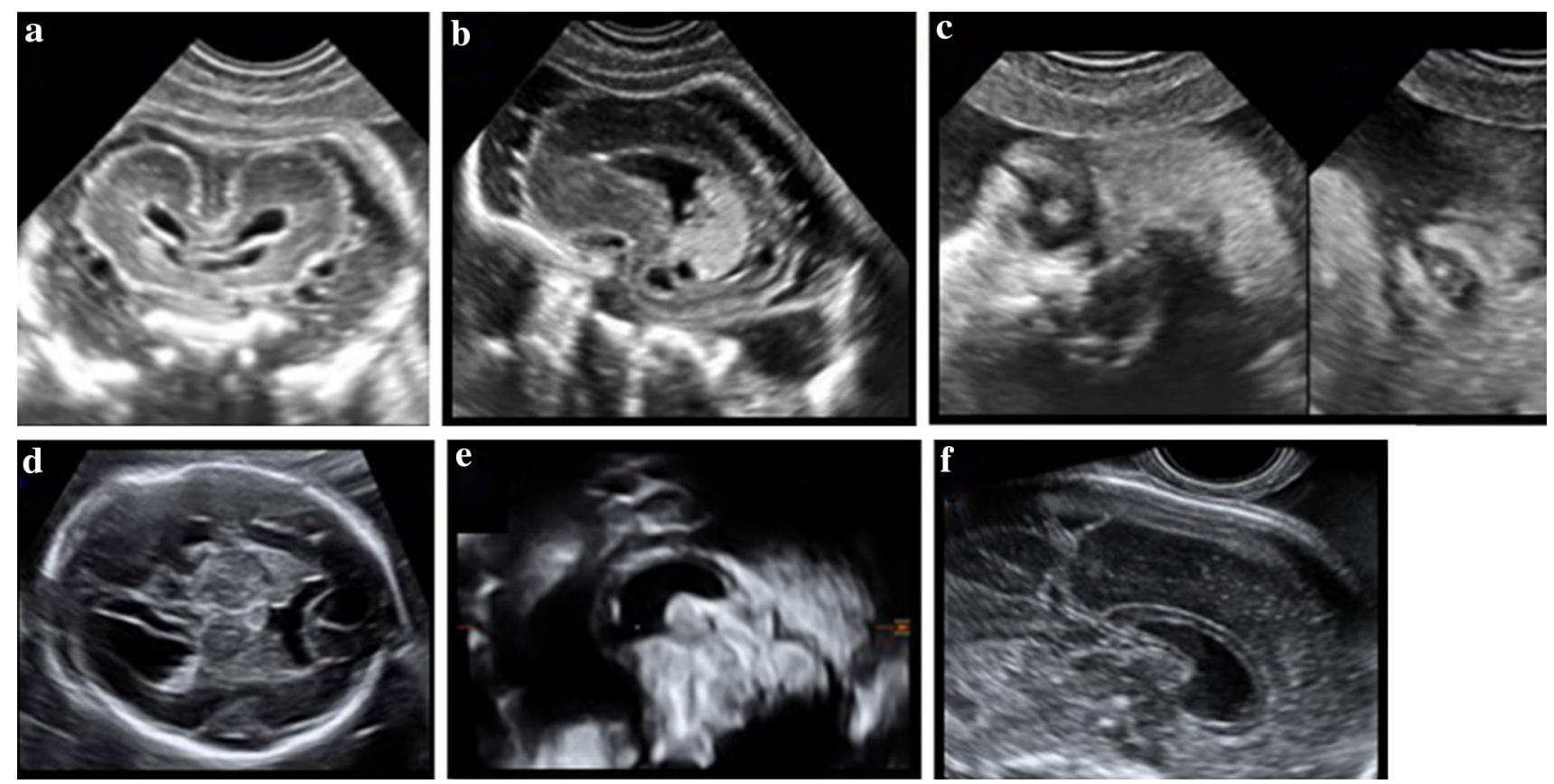

Fig. 2 a Fetal CMV infection, TVS shows enlarged subarachnoid space with intense echos, echogenic ventricular wall with smooth brain and periventricular cystic lesions b Sagittal scan showing periventricular calcification c Cataractous changes and lid edema d Septo-optic dysplasia at 24 weeks of gestation showing nonvisualized cavum septum pellucidum e 3D volume contrast image (VCI) showing corpus callosum $\mathbf{f}$ Sagittal transvaginal section of same case demonstrating all parts of corpus callosum
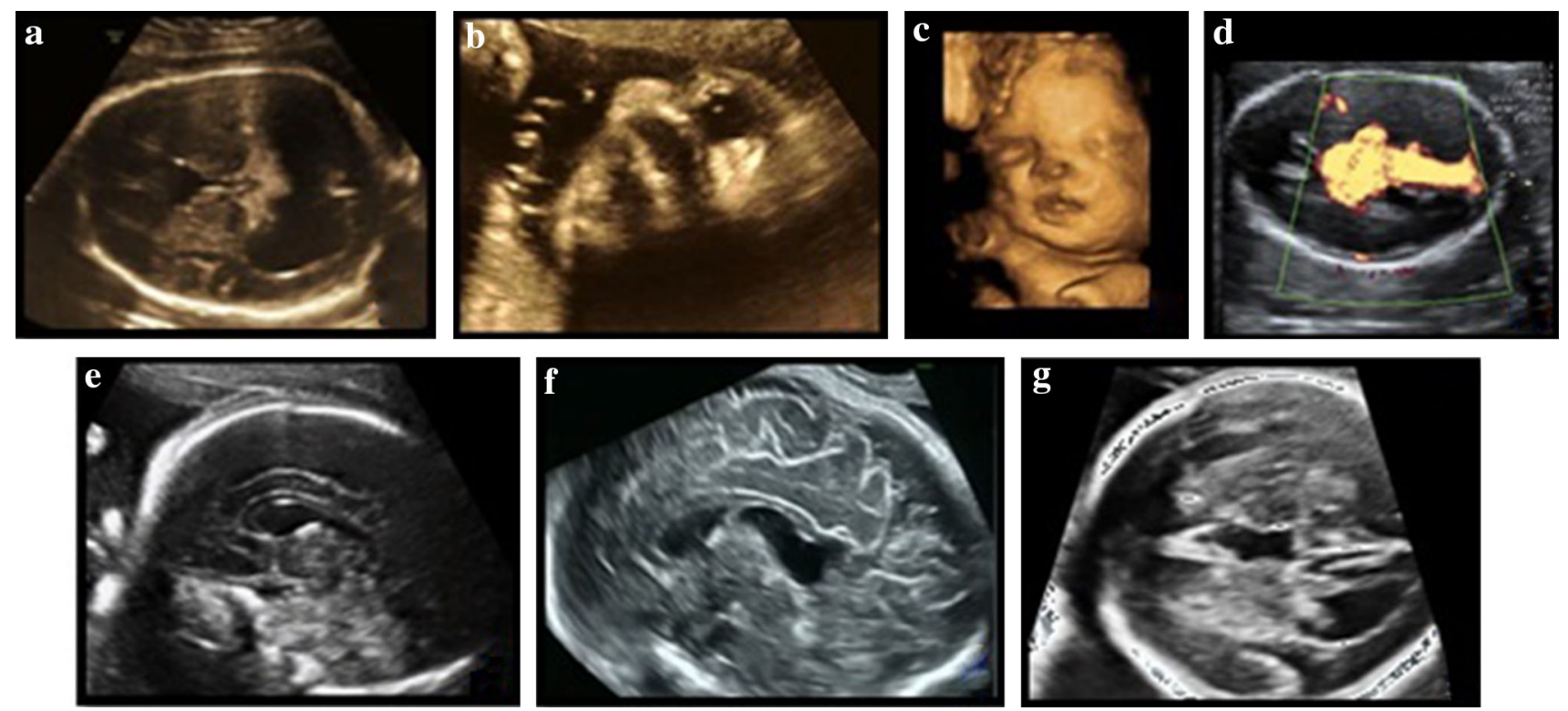

Fig. 3 a 28-weeks-fetus with lobar prosencephaly showing fused, underdeveloped frontal horns and communicating bodies and posterior horns of the lateral ventricles b, $\mathbf{c}$ Unilateral microphthalmia,

horns indicating absence of cavum septum pellucidum. Posteriorly, there was a communication seen between the body of the lateral ventricles-features of lobar holoprosencephaly (Fig. 3a-c).

While looking at the midline cerebral lesions, one should always be aware of the certain normal and abnormal d Vein of Galen malformation, e Cavum vergae, f Cavum veli interpositi, $\mathbf{g}$ Interhemispheric cyst

midline supratentorial cystic lesions. The normal variants of midline cystic lesions are cavum vergae and cavum veli interpositi [10]. The abnormal midline cystic lesions could be arachnoid cyst, vascular malformation, high riding 3rd ventricle and inter-hemispheric cyst associated with agenesis of corpus callosum (Fig. 3d-g). 

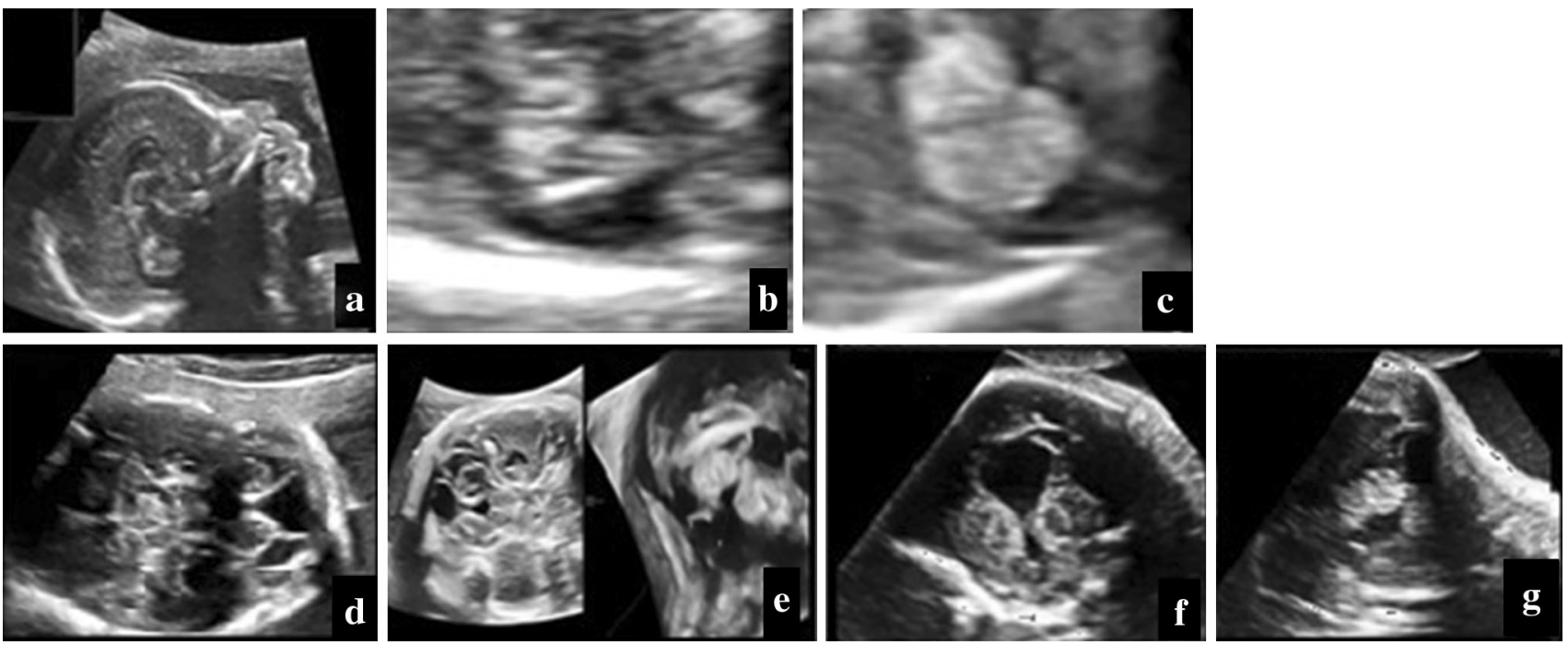

Fig. 4 a Midsagittal view of fetal brain at 24 weeks showing the cerebellar vermis $\mathbf{b}, \mathbf{c} 2 \mathrm{D}$ TAS showing the fastigium and the primary fissure d, e 2D and 3D VCI showing fetal head at 24 weeks with a

In the posterior fossa, the cerebellar vermis was measured supero-inferiorly in the midsagittal plane. Normal vermis should be at least $10 \mathrm{~mm}$ at $18-20$ weeks of gestation. Certain landmarks helped to ascertain the true midsagittal plane. One is the fourth ventricle which is triangular in shape with its beak pointing inferiorly called fastigium. Second is the primary fissure which divides the cerebellar vermis into superior one-third and inferior two-thirds (Fig. 4a-c) [11].

Another case was of a 24-weeks' fetus showing agenesis of corpus callosum with cystic lesion in the posterior fossa. 2D and 3D transabdominal ultrasound showed Blake's pouch cyst communicating with the fourth ventricle. TVS also showed clear visualization of Blake's cyst and cerebellar vermis (Fig. 4d-g). We also had cases of vermian agenesis and Dandy - Walker malformation.

In all cases, the diagnosis could be made by both $2 \mathrm{D}$ and 3D views but 3D added clinically useful information especially in two fetuses with posterior fossa lesions. The 2D median views yielded excellent display of anatomy as well as the abnormalities.

\section{Discussion}

The fetal brain is a structure which evolves continuously throughout gestation. Various structures appear and change their morphology as the pregnancy advances. The guidelines for fetal neurosonography recommend three basic axial planes for assessment of fetal brain. In case of suspicion of any abnormality in these planes, additional planes, namely sagittal and coronal are resorted to. The cystic lesion in the posterior fossa $\mathbf{f}$ Transvaginal scan shows Blake's pouch cyst communicating with the fourth ventricle $\mathbf{g}$ Cerebellar vermis seen

comprehensive evaluation of the midline anomalies of fetal brain involves utilization of all the three planes of imaging to arrive at a conclusive diagnosis. Of these, the sagittal plane is particularly relevant. Obtaining this plane by $2 \mathrm{D}$ ultrasound can be challenging at times due to fetal position. However, 3D ultrasound can come to our rescue in certain situations. The primary disadvantage of 3D ultrasound is the decrease in resolution in the reconstructed planes. TVS can be an effective tool in obtaining high resolution neurosonograms of fetus with a cephalic presentation.

The major midline structures assessed in fetal brain include: falx cerebri, cavum septum pellucidum, corpus callosum, tentorium, cerebellar vermis, fourth ventricle, and cisterna magna. The falx cerebri is seen as an echogenic line in between the cerebral hemispheres. The cavum septum pellucidum has been classically described as a boxlike structure interrupting the falx anteriorly. It is bound by echogenic lines on either side which are in continuity with the medial wall of the lateral ventricle. The corpus callosum is a white matter tract connecting the cerebral hemispheres which appears hypoechoic on sonography. Its parts from anterior to posterior are: rostrum, genu, body, and splenium. At 11-12 weeks' gestation, the corpus callosum begins to develop dorsal to the hippocampal commissure. It grows caudally along with the cerebral hemispheres [12, 13]. By 18 weeks' gestation, all the components of the corpus callosum are present and can be visualized on TVS in approximately $95 \%$ of cases. Because of its relatively late development, various brain anomalies are often associated with abnormalities of the corpus callosum [14]. The tentorium serves as a demarcation between the parts of the 
brain, dividing it into the supratentorial and infratentorial compartments. The cerebellar vermis is seen as an echogenic structure in the posterior fossa with the anechoic fourth ventricle anterior and the cisterna magna posterior to it. The entire vermis and cerebellum are fully formed by the end of the 15th week. However, various studies have reported that the gestational age at which this growth can be appreciated sonographically is much later than the age at which it occurs anatomically. Until the vermis has developed fully, the caudal portions of the fourth ventricle remain covered only by the roof of the fourth ventricle. This roof is sufficiently thin. Therefore, before 16 weeks, separation between the fourth ventricle and the cisterna magna can be difficult to appreciate sonographically [1518].

The present study illustrates the spectrum of midline anomalies of the fetal brain, both supra- and infratentorial. The cases highlight the deviation in morphology from normal of the aforementioned midline structures. The suspicion of these abnormalities arose after imaging the three standard planes. Subsequently, median and coronal views were obtained to arrive at a final diagnosis. It was observed that median views obtained directly with 2D TVS were of superior quality than 3D reconstructed views. Acoustic shadowing from the base of the skull can obscure the brainstem and at times does not allow precise assessment of the relationship between brainstem and the cerebellar vermis when evaluating posterior fossa anomalies. Furthermore, the landmarks of the cerebellar vermis are, frequently, not clearly demonstrated.

In our experience, 2D high resolution TVS is a robust tool in assessment of the supratentorial structures. 3D sonography provides additional information to $2 \mathrm{D}$ ultrasound in the evaluation of the posterior fossa anomalies.

The limitation of the study is the small number of cases. However, this can form the basis of assessment of midline brain anomalies both by $2 \mathrm{D}$ and $3 \mathrm{D}$ ultrasonography in a larger study population and comparison of the two techniques.

Acknowledgments Mangun Kaur assisted in the compilation of data and preparation of manuscript.

\section{Compliance with Ethical Standards}

Conflict of interest None.

\section{References}

1. Sonographic examination of the fetal central nervous system. guidelines for performing the 'basic examination' and the 'fetal neurosonogram'. Ultrasound Obstet Gynecol. 2007;29:109-16.

2. Timor-Tritsch IE, Monteagudo A. Transvaginal fetal neurosonography: standardization of the planes and sections by anatomic landmarks. Ultrasound Obstet Gynecol. 1996;8(1):42-7.

3. Viñals F, Muñoz M, Naveas R, et al. Transfrontal three-dimensional visualization of midline cerebral structures. Ultrasound Obstet Gynecol. 2007;30:162-8.

4. Pilu G, Sandri F, Perolo A, et al. Sonography of fetal agenesis of the corpus callosum: a survey of 35 cases. Ultrasound Obstet Gynecol. 1993;3(5):318-29.

5. Pilu G, Segata M, Ghi T, et al. Diagnosis of midline anomalies of the fetal brain with the three-dimensional median view. Ultrasound Obstet Gynecol. 2006;27(5):522-9.

6. Ghi T, Carletti A, Contro E, et al. Prenatal diagnosis and outcome of partial agenesis and hypoplasia of the corpus callosum. Ultrasound Obstet Gynecol. 2010;35(1):35-41.

7. Nelson MM, Thomson AJ. The acrocallosal syndrome. Am J Med Genet. 1982;12:195-9.

8. Malinger G, Lev D, Zahalka N, et al. Fetal cytomegalovirus infection of the brain: the spectrum of sonographic findings. Am J Neuroradiol. 2003;24(1):28-32.

9. Pilu G, Sandri F, Cerisoli M, et al. Sonographic findings in septooptic dysplasia in the fetus and newborn infant. Am J Perinatol. 1990;7(4):337-9.

10. Epelman M, Daneman A, Blaser SI, et al. Differential diagnosis of intracranial cystic lesions at head US: correlation with CT and MR imaging. Radiographics. 2006;26(1):173-96.

11. Viñals F, Muñoz M, Naveas R, et al. The fetal cerebellar vermis: anatomy and biometric assessment using volume contrast imaging in the C-plane (VCI-C). Ultrasound Obstet Gynecol. 2005;26:622-7.

12. Malinger G, Zakut H. The corpus callosum: normal fetal development as shown by transvaginalsonography. Am J Roentgenol. 1993;161(5):1041-3.

13. Rakic P, Yakovlev PI. Development of the corpus callosum and cavum septi in man. J Comp Neurol. 1968;132:45-72.

14. Barkovich AJ, Norman D. Anomalies of the corpus callosum: correlation with further anomalies of the brain. Am J Roentgenol. 1988;9:493-501.

15. Babcook C, Chong BW, Salamat MS, et al. Sonographic anatomy of the developing cerebellum: normal embryology can resemble pathology. Am J Roentgenol. 1996;166:427-33.

16. Zalel Y, Seidmen DS, Brandt N, et al. The development of the fetal vermis: an in utero sonographic evaluation. Ultrasound Obstet Gynecol. 2002;19:136-9.

17. Bromley B, Nadel AS, Pauker S, et al. Closure of the cerebellar vermis: evaluation with second trimester US. Radiology. 1994;193:761-3.

18. Hashimoto K, Shimizu T, Shimoya K, et al. Fetal cerebellum: US appearance with advancing gestational age. Radiology. $2001 ; 221: 70-4$. 